\title{
Influence of Tumor Subtype, Radiological Sign and Prognostic Factors on Tumor Size Discrepancies Between Digital Breast Tomosynthesis and Final Histology
}

\author{
Alessandro Garlaschi ${ }^{1}$, Massimo Calabrese ${ }^{1}$, Federico Zaottini ${ }^{2}$, Simona Tosto ${ }^{1}$, Marco Gipponi ${ }^{3}$, Paola \\ Baccini $^{4}$, Maurizio Gallo ${ }^{5}$, Alberto Stefano Tagliafico ${ }^{6}$ \\ 1. Radiology, Ospedale Policlinico San Martino, Genova, ITA 2. Radiology, University of Genoa, Genova, ITA 3. Surgery, \\ Ospedale Policlinico San Martino, Genova, ITA 4. Pathology, University of Genova/ AOU IRCCS Policlinico San Martino, \\ Genova, ITA 5. Internal Medicine, University of Genoa, Genova, ITA 6. Radiology, University of Genova/ AOU IRCCS \\ Policlinico San Martino, Genova, ITA
}

Corresponding author: Alberto Stefano Tagliafico, albertotagliafico@gmail.com

\section{Abstract \\ Background}

Influence of tumor subtype, radiological sign and prognostic factors on tumor size discrepancies between DBT and final histology has not been completely investigated so far.

\section{Purpose}

To study the influence of tumor subtype, radiological sign and prognostic factors on tumor size discrepancies between digital breast tomosynthesis and final histology.

\section{Material and methods}

This is a retrospective study conducted between January 2015 and December 2016. After IRB approval, 130 consecutive patients with breast cancer diagnosed with digital breast tomosynthesis (DBT) were evaluated. A discrepancy between DBT and final histology was considered present if the difference was above the cutoff of $5 \mathrm{~mm}$. Tumor subtype, radiological sign and prognostic factors were evaluated in patients with discrepancies. Descriptive statistic and non-parametric tests were used.

\section{Results}

A total of 105 cases of cancer, in 96 patients, all female, were included. Mean age was 61 years (range: 35-82 yrs). In 19 (18.1\%) cases, discrepancies were found: 13 (68.4\%) were underestimated by DBT. For tumor subtype, 10 (52.6\%) were infiltrating lobular carcinomas (ILC) $(\mathrm{p}<0.01)$. Fourteen (73.7\%) discordant cases

Received 07/04/2019 Review began 07/10/2019 Review ended 10/28/2019 Published 10/31/2019

\section{๑) Copyright 2019}

Garlaschi et al. This is an open access article distributed under the terms of the Creative Commons Attribution License CC-BY 3.0., which permits unrestricted use, distribution, and reproduction in any medium, provided the original author and source are credited. were architectural distortions $(\mathrm{p}<0.01)$. Prognostic factors did not affect tumor size discrepancies.

\section{Conclusion}

ILC or an architectural distortion represents the majority of cases of tumor size discrepancies between DBT and final histology.

Categories: Radiology

Keywords: tumor subtype, radiological sign, prognostic factors, digital breast tomosynthesis, size discrepancies

\section{Introduction}

Accurate size prediction of an invasive breast cancer is important in preoperative planning, as a prognostic indicator and consequently for patient management. With the increasing use of breast-conserving surgery and neoadjuvant chemotherapy, the ability to correctly determine maximum tumor extent non-invasively is essential. Under-estimating pre-surgical tumor size may lead to incomplete margins and consequently to reexcision [1]. Although pathologic measurement is regarded as the gold standard, important therapeutic decisions are made on the basis of the tumor size assessment by physical examination and imaging, indeed the pathological tumor staging could not be yet available [1-3].

There have been several attempts to predict breast tumor size based on medical imaging [1-5]. Preoperative tumor size is commonly measured on mammography and ultrasonography. However, using mammography tumor measurements are affected by superimposition of anatomical structures due to the 2D nature of mammography especially at the tumor boundaries [3-5]. Ultrasound, on the other hand, is likely to underestimate the real tumor size due to the overlap in the appearance among desmoplastic reaction and 
tumoral tissue [5]. Digital breast tomosynthesis, a pseudo-3D mammography, could overcome these limitations by reducing the effect of tissue superimposition [1-5]. Digital breast tomosynthesis (DBT) is a three-dimensional X-ray breast imaging method in which high spatial-resolution tomographic images of the breast are reconstructed from multiple low-dose projection exposures acquired by a digital detector from a mammographic X-ray source which moves over a limited angle. The total mean glandular dose for a DBT examination is comparable to that of a two-view mammographic examination, with a higher sensitivity for breast cancer detection [6,7]. DBT and magnetic resonance imaging (MRI) are considered superior to digital mammography and ultrasound (US) in the preoperative assessment of breast tumor size [1] and MRI overestimated tumor size in ductal cancers and slightly underestimated it in lobular cancers [2]. Moreover, DBT showed characteristic imaging features of breast cancers according to molecular subtype [8], but the influence of tumor subtype, radiological sign and prognostic factors on tumor size discrepancies between DBT and final histology has not been completely investigated so far. Therefore, the purpose of this study is to evaluate the influence of tumor subtype, radiological sign and prognostic factors on tumor size discrepancies between digital breast tomosynthesis and final histology.

\section{Materials And Methods}

IRB approval was obtained and written informed consent was waived (Blinded). This is a retrospective study, based on data already stored in the Hospital (Blinded) database. A total of 130 consecutive breast cancer patients (from January 2015 to December 2016) were included. All cancers were visible at DBT and histologically confirmed (all lesions were classified according to the BIRADS including 22 r4b, 24 r4c and 84 r5). Histology was considered suitable for the study if derived from micro-histologic biopsy performed in this center. All cancers were visible at DBT and histologically confirmed. Histology was considered suitable for the study if derived from micro-histologic biopsy performed in this center. Patients operated outside of our center and those cases of small clusters microcalcifications (less than $10 \mathrm{~mm}$ ) studied by vacuum-assisted breast biopsy (VABB) stereotactic or ultrasound-guided were excluded to reduce biases related to patients selection and inaccuracy of measurements for very small lesions. DBT (Hologic, Selenia Dimensions; Bedford, MA) was done with both a mediolateral-oblique (MLO) views and cranio-caudal (CC) views. Two board-certified radiologists with breast imaging experience of 20 and 12 years, respectively, participated in the study. Each reader was blinded to the final results of imaging methods, any clinical information and pathological type of the cancer. Hologic SecurView workstations, optimized to read both 2D and 3D images, were used for screen reading. The longest diameter measured on CC or MLO view was recorded for statistical analysis; the measurements were made on the 3D images. DBT measurements were considered concordant with histology if they were within $\pm 5 \mathrm{~mm}$, and under- and overestimated, respectively, if they were $<5 \mathrm{~mm}$ and $>5 \mathrm{~mm}$ compared to pathological size according to recent literature [1]. In the case of multifocal lesions, it was decided not to consider the extremes of the overall area involved, but to measure each lesion, for the purpose of a more precise evaluation.

\section{Biopsy procedures}

Core-needle biopsy (CB) was done for mass lesions, VABB in cases of micro-calcifications, distortions and asymmetric density. In patients with clusters of microcalcifications, radiographs of biopsy specimens were acquired to document the harvest obtained by means of VABB or $\mathrm{CB}$. Interventional procedures were performed as suggested by recent guidelines [9]. Ultrasound was performed by means of a high-frequency linear-array transducer of $13 \mathrm{MHz}$. In our unit, an automatic spring-loaded $14 \mathrm{G}$ biopsy-needle with a 2/10 $\mathrm{mm}$ penetration depth (Tsunami Medical, Modena, Italy) was used to obtain from three to five cylindershaped samples of tissue from the suspicious area.

\section{Vacuum-assisted breast biopsy}

All VABB procedures were performed with ATEC (Automatic Tissue Extraction and Collection) stereotacticguided breast biopsy system (Hologic, Indianapolis, IN, USA). Twelve cores were taken on a $360^{\circ}$ rotation of the biopsy device and a $9 \mathrm{G}$ needle allowed to remove the lesion in a piecemeal fashion through a 2-3 $\mathrm{mm}$ skin incision. At the end of sampling, the operator inserted a Stereotactic and MR-compatible clip (Atec TriMark, titanium biopsy site marker; Hologic).

\section{Pathological analysis}

Tumor size was assessed by gross tumor and microscopic examination of the excised tumor specimens by one breast pathologist with 22 years of experience in breast pathology. Tumors were classified according to five histological subgroups, including invasive ductal carcinoma (IDC), invasive lobular carcinoma (ILC), ductal carcinoma in situ (DCIS), mixed IDC/ILC and other histological types (mucinous, papillary, medullary, tubular, and apocrine breast carcinoma). According to the common practice of the pathology service of our institution, all surgical specimens were cut into 5-mm thick levels along their longest axis (i.e., 5-mm slicing technique). The measurements were then calculated by multiplying number of levels showing cancer by the thickness of each level. When measuring lesion size only the largest diameter (expressed in $\mathrm{mm}$ ) was considered. In the case of multifocal disease, the final pathology size was obtained by summing the greater microscopic dimension of different tumor foci within the same breast quadrant. 


\section{Cureus}

(PR) status, HER2 expression, Ki-67 proliferation.

\section{Statistical analysis}

Summary statistics were reported for all categorical variables. Mean \pm standard deviation and ranges were reported for numeric variables. Concordance between DBT size and histopathologic size was assessed considering a cut-off rate of $>5 \mathrm{~mm}$ for discrepant cases. Comparisons between concordant and discrepant cases were thus performed using Pearson Chi-square of independence test or Fisher's exact test, as appropriate. Multivariate analysis was used to study prognostic factors in concordant and discordant cases.

\section{Results}

After the exclusion criteria, our series included 105 cases of cancer, in 96 patients, all female (mean age: 61 years; age range: $35-82$ years).

According to radiological findings on DBT there were 37 nodules (35.2\%), 47 distortions (44.8\%) and 21 clusters of microcalcifications (20\%). According to histology, 66 cases (62.8\%) were infiltrating ductal carcinoma, 14 cases (13.3\%) were found to be DCIS, (IDC), 14 cases (13.3\%) infiltrating lobular carcinoma (ILC), and two cases (1.9\%) mixed carcinomas type duct-lobular and finally nine cases $(8.6 \%)$ rare histological types. The average diameter of the lesions at histology was $16.4 \mathrm{~mm} \pm 14.2 \mathrm{~mm}$. Among the most frequent lesions encountered in the study, ductal carcinomas in situ presented in tomosynthesis had an average diameter of $32 \mathrm{~mm}$, infiltrating ductal carcinomas had an average diameter of $13.5 \mathrm{~mm}$, while for infiltrating lobules they had an average diameter of $17 \mathrm{~mm}$.

Significant differences ( $>5 \mathrm{~mm}$ ) between DBT measurement and tumor size at the pathology were present in $19 / 105$ cases (18.1\%). DBT underestimated lesion size in 13/19 (68.4\%) cases and overestimated in 6/19 (31.6\%). Among these 19 neoplastic lesions, the most frequent correspondent radiologic finding at DBT was architectural distortions $(\mathrm{n}=14 / 19 ; 73.7 \%)$. The most represented histological type was infiltrating lobular type ILC $(n=10 / 19,52.6 \%)$, this data was statistically significant at Pearson's Chi-square $(\mathrm{p}<.001)$. The number of discrepancies according to histology is reported in Table 1 and Table 2.

\begin{tabular}{|c|c|c|}
\hline Cancer type & Number of cases & Percentage \\
\hline IDC & 6 & 31.6 \\
\hline DCIS & 0 & 0 \\
\hline ILC & 10 & 52.6 \\
\hline Mixed & 1 & 5.3 \\
\hline Other & 2 & 10.5 \\
\hline
\end{tabular}

\section{TABLE 1: Number and percentage of discrepancies according to histology regardless under- or} over-estimation.

IDC: Invasive ductal carcinoma; DCIS: Ductal carcinoma in situ; ILC: Invasive lobular carcinoma.

\begin{tabular}{|c|c|c|c|c|c|}
\hline & DCIS & IDC & ILC & Mixed carcinomas & Rare histologies \\
\hline Underestimation & 0 & 3 & 8 & 0 & 2 \\
\hline Concordance* $^{*}$ & 14 & 60 & 4 & 1 & 7 \\
\hline Overestimation & 0 & 3 & 2 & 1 & 0 \\
\hline Total & 14 & 66 & 14 & 2 & 9 \\
\hline
\end{tabular}

\section{TABLE 2: Number of discrepancies according to histology.}

${ }^{*}$ Concordance: tumor size discrepancy between digital breast tomosynthesis (DBT) and histology $\leq 5 \mathrm{~mm}$

IDC: Invasive ductal carcinoma; DCIS: Ductal carcinoma in situ; ILC: Invasive lobular carcinoma. 
Number of discrepancies according to radiological sign are reported in Table 3.

\begin{tabular}{|c|c|c|c|}
\hline & Architectural distortions & Masses & Microcalcifications \\
\hline Underestimation & 8 & 4 & 1 \\
\hline Concordance* $^{*}$ & 33 & 33 & 20 \\
\hline Overestimation & 6 & 0 & 0 \\
\hline Total & 47 & 37 & 21 \\
\hline
\end{tabular}

TABLE 3: Number of discrepancies according to radiological sign.

*Concordance: tumor size discrepancy between digital breast tomosynthesis (DBT) and histology $\leq 5 \mathrm{~mm}$.

Comparing the overall distribution of tumor grading among cases without measurement discrepancies (G1 n $=21(20.0 \%) ; \mathrm{G} 2 \mathrm{n}=71(67.6 \%) ; \mathrm{G} 3, \mathrm{n}=13(12.4 \%))$ and the grading distribution among tumors with underor overstimated size (G1 n = 5 (26.3\%), G2 n=12 (63.2\%), and G3 n = $2(10.5 \%)$ ) there were no significant differences $(p<0.05)$. Similarly, there were no significant differences $(p<0.05)$ for estrogen receptor $(E R)$ and progesterone receptor (PR) status, HER2 expression, Ki-67 proliferation.

\section{Discussion}

Accurate measurement of tumor extent is important for tumor staging, which in turn is an important parameter making decision on treatment [3]. Recent literature strongly suggests that DBT is superior to digital mammography for breast cancer detection; therefore DBT usage is increasing in clinical practice in screening and diagnostic settings [10-16]. By consequence, breast cancer measurements will likely frequently occur directly on DBT. For this reason, in this study, we evaluated the influence of tumor subtype, radiological sign and prognostic factors on tumor size discrepancies between digital breast tomosynthesis and final histology. The results of this study found that the majority of cases of tumor size discrepancies between DBT and final histology are recorded when the tumor is an ILC at final histology or when the radiological sign is an architectural distortion at DBT. Indeed, in 19 cancers with size discrepancies, 10 (52.6\%) were infiltrating lobular carcinomas (ILC) and 14 (73.7\%) discordant cases were architectural distortions on DBT. In addition, DBT resulted to underestimate cancer size in the majority of cases $(\mathrm{n}=$ $13 / 19,68.4 \%)$. When compared with the data available in the literature, the percentage of discrepancy between tomosynthesis and histology, using a predetermined 5-mm cut-off, resulted to be between $10 \%$ $20 \%$ [1]. The relatively large number of architectural distortions that determined tumor size discrepancies could be interpreted as the difficulty in correct measuring a cancer presenting as a distortions in DBT. Indeed, despite the attempt to estimate only the core of the lesion excluding as much as possible the spicules, lesion borders probably remained unclear. Moreover, although it is shown that even for invasive lobular carcinomas, the sensitivity of the DBT is greater than that of digital mammograpy [17], this study suggests a reduced reliability in correctly defining the actual size of the tumor. Our results are concordant with a recent study in which DBT failed to accurately assess tumor size of ILC and concordance in tumor size measurement between DBT and pathology was fair (intraclass correlation coefficient $=0.24$ ) [17]. It is possible that the coexistence of infiltrating lobular carcinoma at histology and architectural parenchimal distortion at imaging finding represent the combination with greater risk of discrepancies in the exact definition of tumor size. Indeed, invasive lobular carcinoma (ILC) is the most common "special” type of breast cancer (approximately $5-15 \%$ of all breast cancers) with a characteristic grow pattern involving the infiltration of single cells or single files of cells through the stroma, with little alterations of normal tissue architecture and relative preservation of normal breast parenchymal architecture [18]. The complex biology of ILC is probably one of the possible explanations of tumor size discrepancies among DBT and final histology. This data regarding DBT measurements could be considered consistent with the recent observation on MRI that tumor type and poorly defined margins are independent factor for imagingpathology discordance in lesion sizing [19].

Regarding common prognostic factors, no significant differences were found among patients with concordance and discrepancies among DBT and final histology for grading, estrogen receptor and progesterone receptor, HER2 expression and Ki-67 proliferation. This data could suggest that common prognostic factors do not influence the rate of disagreement between DBT and pathology examination for tumor size estimation. However, it is possible that more complex analysis on prognostic factors such as those based on radiomics will find some prognostic features linked to tumor size discrepancies [20-22].

This study has several limitations. The first is the retrospective nature, the second is that we have not considered other diagnostic tools potentially more sensitive than tomosynthesis, such as the MRI. However, DBT usage is likely to become largely adopted in screening and diagnostic setting whereas MRI is limited to 
specific indications. In addition, the sample of women included in the study could be considered not so large to detect small differences especially regarding prognostic factors. However the sample size was deemed to be sufficient to detect differences if clinically relevant. Moreover, the number of patients was not sufficient to compare rare tumoral subtypes, but the data of this study could be included in future meta-analyses. Finally, we did not evaluate advanced imaging features to be correlated with common prognostic factors. Indeed, it has been shown that some radiomic features can be distinguishing between ER+ versus ER-, PR+ versus PR-, HER2+ versus HER2-, and triple-negative adding new insights into breast cancer imaging [21]. Further studies are needed to assess if some radiomic features with potential prognostic value could influence tumor size discrepancies among imaging techniques and final histology.

\section{Conclusions}

DBT showed characteristic imaging features of breast cancers according to molecular subtype, however, the influence of tumor subtype, radiological sign and prognostic factors on tumor size discrepancies between DBT and final histology has not been completely investigated so far and we evaluated the influence of tumor subtype, radiological sign and prognostic factors on tumor size discrepancies between DBT and final histology on 105 cases of cancer in 96 patients. This study found that tumor size discrepancies between DBT and final histology are mainly due to the diagnosis of ILC at histology or to an architectural distortion seen on DBT. Common prognostic factors did not affect tumor size discrepancies.

\section{Additional Information}

\section{Disclosures}

Human subjects: Consent was obtained by all participants in this study. IRB approval was obtained and written informed consent was waived issued approval 263REG2017. IRB approval was obtained and written informed consent was waived (263REG2017). This is a retrospective study, based on data already stored in the Hospital database. Animal subjects: All authors have confirmed that this study did not involve animal subjects or tissue. Conflicts of interest: In compliance with the ICMJE uniform disclosure form, all authors declare the following: Payment/services info: All authors have declared that no financial support was received from any organization for the submitted work. Financial relationships: All authors have declared that they have no financial relationships at present or within the previous three years with any organizations that might have an interest in the submitted work. Other relationships: All authors have declared that there are no other relationships or activities that could appear to have influenced the submitted work.

\section{References}

1. Luparia A, Mariscotti G, Durando M, et al.: Accuracy of tumour size assessment in the preoperative staging of breast cancer: comparison of digital mammography, tomosynthesis, ultrasound and MRI. Radiol Med. 2013, 118:1119-1136. 10.1007/s11547-013-0941-Z

2. Leddy R, Irshad A, Metcalfe A, Mabalam P, Abid A, Ackerman S, Lewis M: Comparative accuracy of preoperative tumor size assessment on mammography, sonography, and MRI: is the accuracy affected by breast density or cancer subtype?. J Clin Ultrasound. 2016, 44:17-25. 10.1002/jcu.22290

3. Dummin LJ, Cox M, Plant L: Prediction of breast tumor size by mammography and sonography--A breast screen experience. Breast. 2007, 16:38-46. 10.1016/j.breast.2006.04.003

4. Wasif N, Garreau J, Terando A, Kirsch D, Mund DF, Giuliano AE: MRI versus ultrasonography and mammography for preoperative assessment of breast cancer. Am Surg. 2009, 75:970-975.

5. Förnvik D, Zackrisson S, Ljungberg O, Svahn T, Timberg P, Tingberg A, Andersson I: Breast tomosynthesis: accuracy of tumor measurement compared with digital mammography and ultrasonography. Acta Radiol. 2010, 51:240-247. 10.3109/02841850903524447

6. Endo T, Morita T, Oiwa M, et al.: Diagnostic performance of digital breast tomosynthesis and full-field digital mammography with new reconstruction and new processing for dose reduction. Breast Cancer. 2018, 25:159-166. 10.1007/s12282-017-0805-9

7. Mariscotti G, Durando M, Houssami N, et al.: Comparison of synthetic mammography, reconstructed from digital breast tomosynthesis, and digital mammography: evaluation of lesion conspicuity and BI-RADS assessment categories. Breast Cancer Res Treat. 2017, 166:765-773. 10.1007/s10549-017-4458-3

8. Lee SH, Chang JM, Shin SU, Chu AJ, Yi A, Cho N, Moon WK: Imaging features of breast cancers on digital breast tomosynthesis according to molecular subtype: association with breast cancer detection. British J Radiol. 2017, $27: 20170470.10 .1259$ /bjr.20170470

9. van Dongen JA, Bartelink H, Fentiman IS, et al.: Factors influencing local relapse and survival and results of salvage treatment after breast-conserving therapy in operable breast cancer: EORTC trial 10801, breast conservation compared with mastectomy in TNM stage I and II breast cancer. Eur J Cancer. 1992, 28A:801805. 10.1016/0959-8049(92)90118-1

10. Houssami N, Lång K, Bernardi D, Tagliafico A, Zackrisson S, Skaane P: Digital breast tomosynthesis (3Dmammography) screening: a pictorial review of screen-detected cancers and false recalls attributed to tomosynthesis in prospective screening trials. Breast. 2016, 26:119-134. 10.1016/j.breast.2016.01.007

11. Bernardi D, Belli P, Benelli E, et al.: Digital breast tomosynthesis (DBT): recommendations from the Italian College of Breast Radiologists (ICBR) by the Italian Society of Medical Radiology (SIRM) and the Italian Group for Mammography Screening (GISMa). Radiol Med. 2017, 122:723-730. 10.1007/s11547-017-0769-z

12. Bernardi D, Macaskill P, Pellegrini M, et al.: Breast cancer screening with tomosynthesis (3D mammography) with acquired or synthetic 2D mammography compared with 2D mammography alone (STORM- 2): a 
population-based prospective study. Lancet Oncol. 2016, 17:1105-1113. 10.1016/S1470-2045(16)30101-2

13. Tagliafico A, Houssami N, Calabrese M: Digital Breast Tomosynthesis: A Practical Approach. Springer International Publishing, 2016. 10.1007/978-3-319-28631-0

14. Tagliafico AS, Mariscotti G, Valdora F, et al.: A prospective comparative trial of adjunct screening with tomosynthesis or ultrasound in women with mammography-negative dense breasts (ASTOUND-2). Eur J Cancer. 2018, 104:39-46. 10.1016/j.ejca.2018.08.029

15. Tagliafico AS, Calabrese M, Bignotti B, et al.: Accuracy and reading time for six strategies using digital breast tomosynthesis in women with mammographically negative dense breasts. Eur Radiol. 2017, 27:51795184. 10.1007/s00330-017-4918-5

16. Gilbert FJ, Tucker L, Gillan MG, et al.: TOMMY trial: a comparison of TOMosynthesis with digital MammographY in the UK NHS Breast Screening Programme. Health Technol Assess. 2015, 19:1-136. 10.3310/hta19040

17. Chamming's F, Kao E, Aldis A, Ferré R, Omeroglu A, Reinhold C, Mesurolle B: Imaging features and conspicuity of invasive lobular carcinomas on digital breast tomosynthesis. British J Radiol. 2017, 90:20170128. 10.1259/bjr.20170128

18. Oliveira TMG, Elias J, Melo AF, et al.: Evolving concepts in breast lobular neoplasia and invasive lobular carcinoma, and their impact on imaging methods. Insights Imaging. 2014, 5:183-194. 10.1007/s13244-0140324-6

19. Mennella S, Garlaschi A, Paparo F, et al.: Magnetic resonance imaging of breast cancer: factors affecting the accuracy of preoperative lesion sizing. Acta Radiol. 2015, 56:260-268. 10.1177/0284185114524089

20. Gillies RJ, Kinahan PE, Hricak H: Radiomics: images are more than pictures, they are data . Radiology. 2016, 20:563-577. 10.1148/radiol.2015151169

21. Tagliafico AS, Valdora F, Mariscotti G, et al.: An exploratory radiomics analysis on digital breast tomosynthesis in women with mammographically negative dense breasts. Breast. 2018, 40:92-96. 10.1016/j.breast.2018.04.016

22. Limkin EJ, Sun R, Dercle L, et al.: Promises and challenges for the implementation of computational medical imaging (radiomics) in oncology. Ann Oncol. 2017, 28:1191-1206. 10.1093/annonc/mdx034 